\title{
NT-MDT - Integrated Solutions for Nanotechnology
}

\author{
I. Mościcki, G. KASZYŃSKI \\ IGHT s.c., Poland, www.ight.com.pl; NT-MDT — www.ntmdt.com
}

NT-MDT is a global company that provides integrated solutions for nanotechnology. In this article we would like to present one of the most advanced systems NTEGRA Spectra - with an example of application graphene characterization. For more details about systems and other applications please contact us or visit our webpage www.ntmdt.com .

\section{Introduction}

The interest in graphene is continuously rising among science communities. Applied physicists, new material designers, and nanotechnology engineers are attracted by its unique properties such as electrical and thermal conductivity. Fundamental physicists are enchanted by the possibility of exploring quantum relativistic phenomena on 2-dimensional crystals that have a unusual electronic spectrum. In order to manipulate a single-atom-thin structure, one needs a reliable high precision tool.

In this article two experimental examples are presented. The first one shows the advantages of the NTEGRA based atomic force microscopy (AFM) and how it can reveal the fine structure of graphene flakes. The second example shows how the NTEGRA combines scanning probe microscopy (SPM) with the Raman spectroscopy to become a NanoLaboratory with advanced analytical capability.

\section{AFM experiments}

Scheme of the layers is shown in the figure.

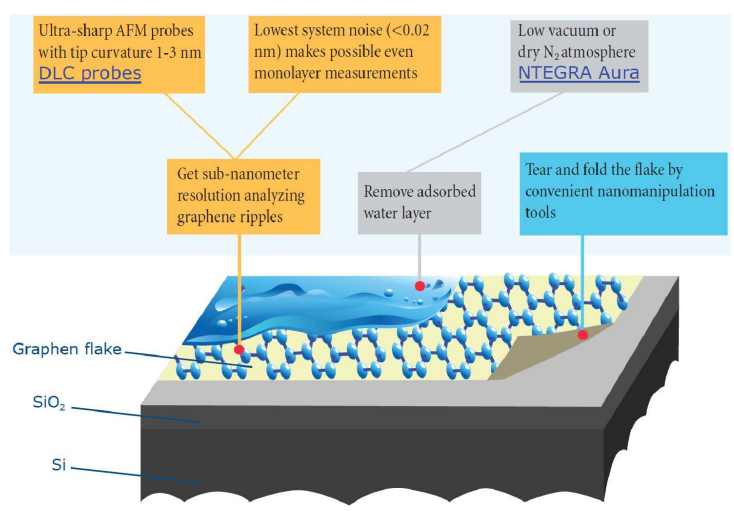

\section{Complex experiment: Raman spectroscopy + SPM NanoLaboratory NTEGRA spectra}

The powerful analytical capabilities of scanning probe microscopy and Raman microscopy have been successfully integrated with NTEGRA Spectra NanoLaboratory.

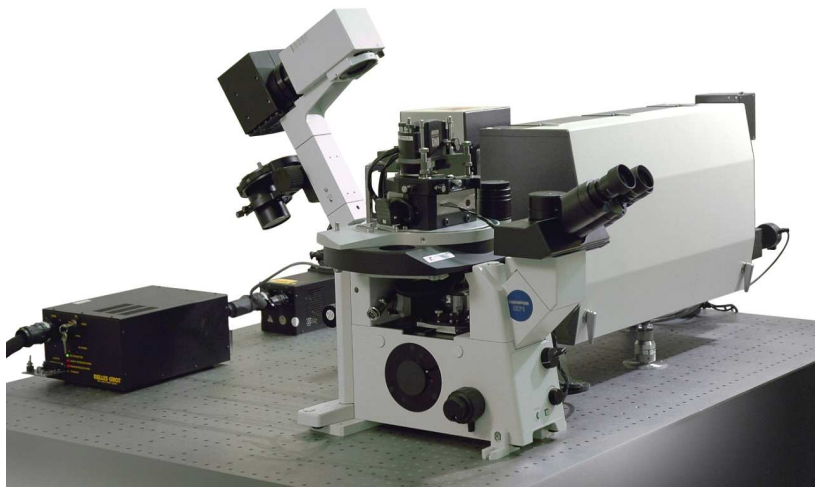

The NTEGRA Spectra provides a comprehensive characterization of the graphene specimen. Shown are images and quantitative data (the figure on the next page) obtained from the same graphene sample (placed on $\mathrm{Si} / \mathrm{SiO}_{2}$ substrate) obtained in a single experiment using the AFM - Raman setup [1]. (a) White light image of multi-layer graphene sample obtained with high resolution $100 \times, 0.7$ NA objective. 1-, 2-, 3-, and 4-layered flakes are observed. Image size $120 \times 110 \mu \mathrm{m}$. (b) AFM topography of the same sample with corresponding line profiles. Scan size $50 \times 50 \mu \mathrm{m}$. (c) Raman spectra of graphene flakes. 2D $\left(G^{\prime}\right)$ Raman peak changes in shape width and position for an increasing number of layers reflecting a change in electron band structure. (d) Confocal Raman map (2D band center of mass position). 1-, 2-, 3 -, and 4-layered flakes can be easily distinguished when using a color palette scale. (e) and (f) Scanning Kelvin probe microscopy of negatively and positively charged graphene. The flakes were charged by applying $+3 \mathrm{~V}$ (e) or $-3 \mathrm{~V}$ (f) voltage with conductive cantilever. Scan size $90 \times 80 \mu \mathrm{m}$. (g) Electrostatic force microscopy of the same sample. Scan size $65 \times 55 \mu \mathrm{m}$. (h) Scanning capacitance microscopy image. Singularity in capacitance distribution can be seen at the edge of the layer reflecting graphene electron edge states. Scan size $25 \times 25 \mu \mathrm{m}$. 


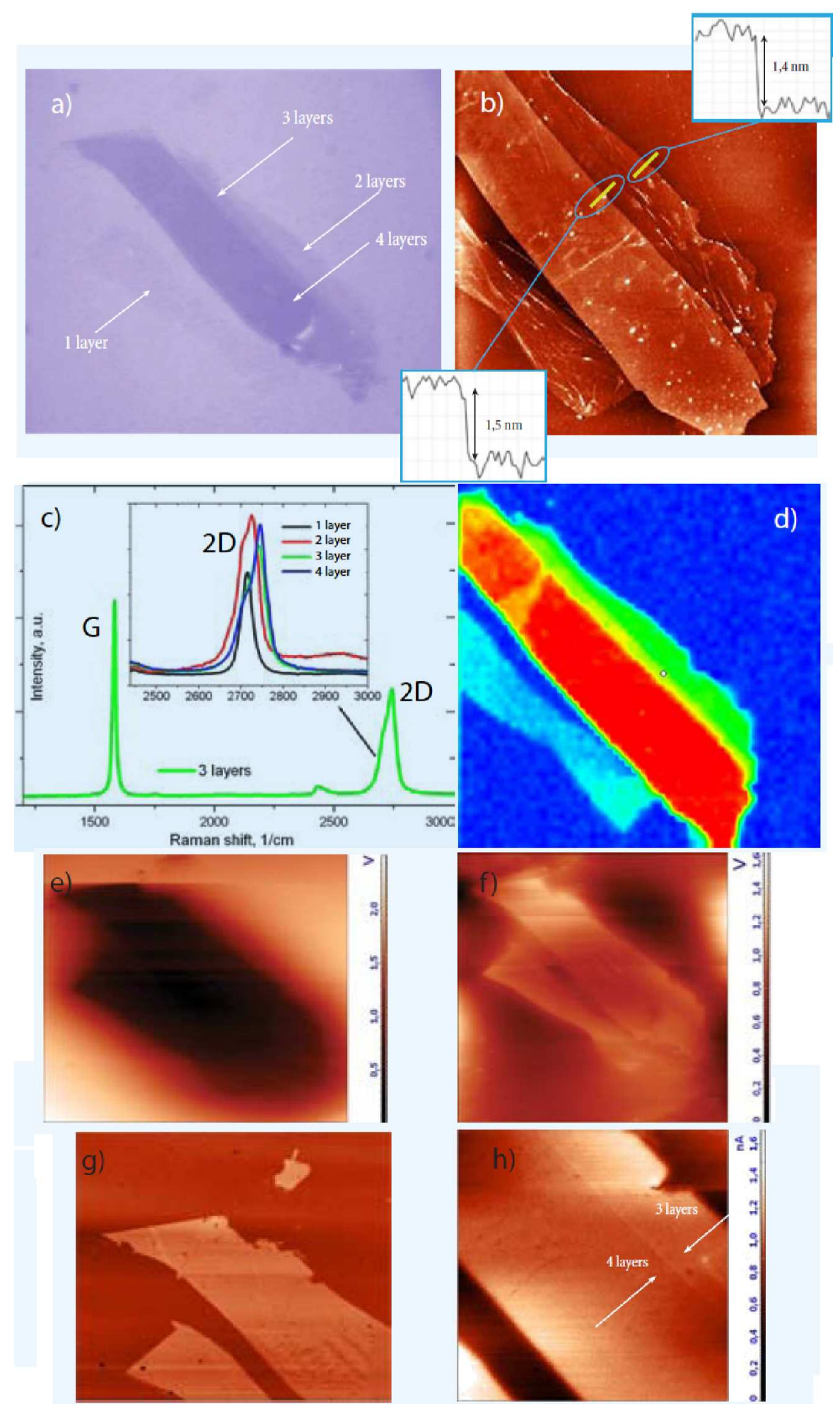

\section{References}

[1] Details of original experiment, see F.V. Tikhonenko, D.W. Horsell, R.V. Gorbachev, A.K. Savchenko, Phys. Rev. Lett. 100, 056802 (2008). 